\title{
Psychometric Properties of the Communication Confidence Rating Scale for Aphasia (CCRSA): Phase 2
}

\section{Background}

Given the impetus from national and international organizations, clinicians need to have access to tools to fully assess how well the person is living with aphasia (Kagan \& Simmon-Mackie, 2007). Communication confidence is one construct that may be related to quality of life, but has not been explored in aphasia research until recently (reference removed for anonymity). The construct of communication confidence was introduced by participants and family members during qualitative post-treatment interviews as part of a research study using a computer program to deliver language therapy (reference removed for anonymity). However, communication confidence was not assessed fully in the standardized evaluation protocol. Only one test, the ASHA-QCL, touched on communication confidence by asking the person to rate a single item "I am confident I can communicate.” Therefore, a comprehensive scale asking persons to self-rate communication confidence was needed.

This poster presents data from the second phase of a project in which a self rating scale of communication confidence has been developed and tested. In phase 1, an 8-item CCRSA, based on items from the ASHA-QCL, was developed and administered 42 times (reference removed for anonymity). Although Rasch analysis indicated good person and item reliability, recommendations for improvement were made to modify the questionnaire. In Phase 2, a revised 10-item CCRSA has been evaluated psychometrically.

\section{Method}

Development of the CCRSA

Phase 1

The Communication Confidence Rating Scale for Aphasia (CCRSA) was based on eight items from the ASHA-QCL which were well-suited to being adapted to rating confidence. The items were reworded in a format similar to a questionnaire in the stuttering literature, the Self-Efficacy Scaling for Adult Stutters (SESAS- Ornstein \& Manning, 1985), which asks participants to rate confidence in a variety of communication situations on a scale from 0-100. See Table 1 for the CCRSA items developed from the ASHA-QCL.

\section{Phase 2 - Revised CCRSA:}

Recommendations to improve person and item reliability resulting from the previous Rasch analysis (reference removed for anonymity) were to (1) divide one item which addressed two different communication situations into two items, and (2) include an item which would address more challenging communication situations for persons with milder aphasia. The result was a 10item questionnaire. See Table 2 for the revised 10-item CCRSA. 
The following is an example from the CCRSA:

How confident are you about your ability to talk with people?

\begin{tabular}{lcccccccccc}
0 & 10 & 20 & 30 & 40 & 50 & 60 & 70 & 80 & 90 & 100 \\
1 & 1 & 1 & 1 & 1 & 1 & 1 & 1 & 1 & 1 & 1 \\
\hline $\begin{array}{c}\text { Not } \\
\text { Confident }\end{array}$ & \multicolumn{1}{c}{$\begin{array}{c}\text { Moderately } \\
\text { Confident }\end{array}$}
\end{tabular}

Subjects

The CCRSA was administered to 75 male and 19 female PWAs. Seventy were Caucasian, 12 were African American, 9 were Hispanic, and 3 were Asian. Eighty-nine PWAs were right handed prior to their stroke, and five were left handed. Participant's severity of aphasia was determined by WAB-AQ scores which ranged from 10.9 to 94.9 with a mean of 56.8 ( $\mathrm{SD}=23.48)$. Seventy-two PWAs presented with non-fluent aphasia and 22 with fluent aphasia. Mean age at time of administration was 57.9 years $(\mathrm{SD}=10.9$; range $=23.8$ to 75.3$)$. The average time post-onset to date of administration was 40 months $(\mathrm{SD}=48.6$; range=2.3 to 190.4). Mean education level was 16.1 years $(\mathrm{SD}=3.37$; range $=10$ to 23 years $)$.

\section{Administration}

Participants completed the CCRSA as part of a research protocol or as participants in community aphasia groups. All subjects completed the CCRSA within one week of WAB-AQ testing. Questions were read aloud by a speech-language pathologist at the same time as the PWAs were able to see the questions and the rating scale in front of them.

\section{$\underline{\text { Results }}$}

The mean score of the 94 administrations was $64.5 / 100$ ( $\mathrm{SD}=17.48$; range=21-99). The interquartile range was 55 to 77.9. PWAs in the $1^{\text {st }}$ quartile had a mean WAB AQ of 44.63 $(\mathrm{SD}=21.92)$ versus a mean WAB AQ of 65.31 ( $\mathrm{SD}=25.8)$ for PWAs in the $4^{\text {th }}$ quartile. Therefore, severity of aphasia may impact a person's communication confidence. Because the CCRSA uses an ordinal rating scale, Rasch analysis is underway to provide additional information about person-item difficulty and fit (Rasch, 1960; Linacre, 2008; Wright \& Masters, 1982).

\section{$\underline{\text { Discussion and Conclusions }}$}

Communication confidence is not typically assessed in formal aphasia evaluations and may be an important construct to explore. Preliminary results from statistical analysis of the CCRSA suggest that it is a potentially useful tool to evaluate communication confidence in persons with aphasia. Data collected from 94 administrations indicate severity of aphasia may impact perceived communication confidence. Generally, persons with more severe aphasia, as indicated by lower WAB-AQ scores, demonstrated lower communication confidence ratings. However, some individuals with relatively mild aphasia also demonstrated decreased confidence in communicating. The CCRSA may be one way to identify communication situations that are challenging to the PWA which then be addressed in a therapeutic setting. 


\section{$\underline{\text { Research Implications }}$}

Communication confidence is an important construct to assess. Severity of aphasia may impact a person's confidence in communicating in different situations; however, there may be other factors that impact communication confidence, and this requires further exploration. Although Rasch analysis provides information about person-item difficulty and fit, future evaluation of the psychometric properties of the CCRSA should focus on internal and external validity and interand intra-rater reliability.

Funding for the study was provided by US Department of Education, National Institute on Disability and Rehabilitation Research through Grant \# H133G060055 and Grant \# H133B031127 (Rehabilitation Research and Training Center on Technology Promoting Integration for Stroke Survivors: Overcoming Societal Barriers).

\section{References}

Chapey R, Duchan JF, Elman RJ, Garcia LJ, Kagan A, Lyon J, Simmons-Mackie, N. Life Participation Approach to Aphasia: A Statement of Values for the Future. Retrieved April 5, 2009: American Speech-Language and Hearing Association website: http://www.asha.org/public/speech/disorders/LPAA.htm

Kagan A, Simmons-Mackie N. Beginning with the end: Outcome-driven assessment and intervention with life participation in mind. Top Lang Disorders. 2007;4:309-317.

Linacre, J.M. Winsteps version 3.65.0 4/3/2008, PO Box 811322, Chicago IL 60681-1322, USA.

Ornstein, A.F., and Manning, W.H. (1985). Self-Efficacy Scaling by Adult Stutters. Journal of Communication Disorders. 18(1985). 313-320.

Rasch, G. (1960). Probabilistic models for some intelligence and attainment tests. Copenhagen: Danmarks Paedogogiske Institut 1960 (Chicago: University of Chicago Press 1980).

Wright, B. D. \& Masters, G. (1982). Rating scale analysis: Rasch measurement. Chicago: MESA Press. 
Table 1 - Questions from ASHA-QCL developed for the CCRSA ASHA QCL Communication Confidence Rating Scale for

Aphasia

\begin{tabular}{|l|l|}
\hline 1. I like to talk with people. & $\begin{array}{l}\text { 1. How confident are you about your ability to talk with } \\
\text { people? }\end{array}$ \\
\hline 6. I stay in touch with family and friends. & $\begin{array}{l}\text { 2. How confident are you about your ability to stay in touch } \\
\text { with family and friends? }\end{array}$ \\
\hline 7. People include me in conversations. & $\begin{array}{l}\text { 3. How confident are you that people include you in } \\
\text { conversations? }\end{array}$ \\
\hline $\begin{array}{l}\text { 8. I follow news, sports, and stories on } \\
\text { TV/movies. }\end{array}$ & $\begin{array}{l}\text { 4. How confident are you about your ability to follow news, } \\
\text { sports, and stories on TV/movies? }\end{array}$ \\
\hline 9. I use the telephone. & $\begin{array}{l}\text { 5. How confident are you about your ability to speak on the } \\
\text { telephone? }\end{array}$ \\
\hline 11. People understand me when I talk. & $\begin{array}{l}\text { 6. How confident are you that people understand you when } \\
\text { you talk? }\end{array}$ \\
\hline 13. I make my own decisions. & $\begin{array}{l}\text { 7. How confident are you that you can make your own } \\
\text { decisions? }\end{array}$ \\
\hline 17. I speak for myself. & $\begin{array}{l}\text { 8. How confident are you about your ability to speak for } \\
\text { yourself? }\end{array}$ \\
\hline
\end{tabular}

Table 2 - Revised CCRSA Questions

Original Questionnaire

1. How confident are you about your ability to talk with people?

2. How confident are you about your ability to stay in touch with family and friends?

3. How confident are you that people include you in conversations?

4. How confident are you about your ability to follow news, sports, and stories on TV/movies?

Revised Questionnaire

1. Same

2. Same

3. Same

4. How confident are you about your ability to follow news and sports on TV?

5. How confident are you about your ability to follow movies on TV or in a theater?

5. How confident are you about your ability to speak on the telephone?

6. How confident are you that people understand you when you talk?

7. How confident are you that you can make your own decisions?

8. How confident are you about your ability to speak for yourself?

6. Same

7. Same

8. Same

9. Same

10. How confident are you that you can participate in conversations about your finances? 